\title{
Germinal matrix hemorrhage-intraventricular hemorrhage: pathogenesis and outcomes
}

\author{
Ettore Piro \\ From XXI Congress of the Italian Society of Neonatology \\ Palermo, Italy. 24-26 September 2015
}

Germinal matrix hemorrhage-intraventricular hemorrhage (GMH-IVH) is one of the CNS injuries affecting preterm infants occurring in about $15 \%-20 \%$ of subjects weighing less than $1500 \mathrm{~g}$. Currently, using ultrasonography, we recognize three grades of GMH-IVH. Grade I involving the subependymal parenchyma and/or extending in less than $10 \%$ of the ventricle, grade II with intraventricular bleeding not expanding in more than $50 \%$ of the ventricle, grade III characterized by consistent (> 50\%)intraventricular bleeding with ventricular dilatation. A concomitant intraparenchymal lesion (IPL), due to a venous infarction (ex grade IV), can be associated with any grade of IVH, worsening the prognosis.

Pathogenesis of GMH-IVH is multifactorial and complex, due to several factors identified as genetic predisposition, systemic (cardiorespiratory, hematologic, immunologic and metabolic) and developmental (immature anatomical substrates and immature, impaired cerebrovascular reactivity of the preterm brain) predisposing to intraventricular bleeding. Prenatal chorioamnionitis with umbilical vasculitis, primarily due to Ureaplasma species, is considered the main prenatal factor responsible for increased risk of GMH-IVH [1,3].

Of primary importance for the occurrence of intraventricular bleeding is considered the hemodynamic instability that, in the first three days of life, affects the extreme preterm, in which cerebral vasoreactivity and autoregolatory mechanisms to pressure variability are weak. In extremely preterm newborns, hypercapnia $(\mathrm{paCo} 2>55$ $\mathrm{mmHg}$ ), with consequent cerebral vasodilatation, arterial hypotension and persistent patent ductus arteriosus with diastolic steal, are responsible for the cerebral blood flow instability. Neonatal intubation, ventilatory strategies,

Correspondence: ettore.piro@unipa.it

DepartmentofSciencesforHealth Promotion andMother-ChildCare "G. D'Alessandro", University of Palermo, Italy

C 2015 Piro This is an Open Access article distributed under the terms of the Creative Commons Attribution License (http:// creativecommons.org/licenses/by/4.0), which permits unrestricted use, distribution, and reproduction in any medium, provided the original work is properly cited. The Creative Commons Public Domain Dedication waiver (http://creativecommons.org/publicdomain/ zero/1.0/) applies to the data made available in this article, unless otherwise stated. intravenous fluid and electrolytes management and neonatal complications also play an important role.

In about $35 \%$ of infants with intraventricular bleeding a posthemorragic ventricular dilatation (PHVD) will occur, with possible evolution in about $22 \%$ in posthemorrhagic hydrocephalus $(\mathrm{PHH})$, that in about $9 \%$ will require the placement of a permanent shunt, therefore complicating the outcomes [2]. Of primary importance is the treatment of symptomatic PHH. Communicanting and non communicanting hydrocephalus have different treatment options. Some preterm infants may be affected by a transient symptomatic form, and therefore need a short period of CSF diversion. In communicating hydrocephalus serial lumbar puncture, better if performed as "early intervention", are associated with a reduced need of persistent shunt insertion. Ventriculoperitoneal shunt is the more frequent form of permanent CSF diversion used in PHH. Infections and shunt revisions are its main complications. In non communicating hydrocephalus endoscopic third ventriculostomy is an important alternative [3]. Survivors can be affected by neurological, neurosensorial, cognitive and behavioral impairment depending from the individual risk profile.

\section{Published: 24 September 2015}

\section{References}

1. O'Leary H, Gregas MC, Limperopoulos C, Zaretskaya I, Bassan H, Soul JS, et al: Elevatedcerebral pressure passivity is associated with prematurityrelated intracranial hemorrhage. Pediatrics 2009, 124:302-309.

2. Vassilyadi M, Tataryn Z, Shamji MF, Ventureyra EC: Functional outcomes among prematureinfants with intraventricular hemorrhage. PediatrNeurosurg 2009, 45:247-255.

3. Robinson S: Neonatal posthemorrhagic hydrocephalus from prematurity: pathophysiology and current treatment concepts. J NeurosurgPediatr 2012, 9(3):242-58.

doi:10.1186/1824-7288-41-S1-A31

Cite this article as: Piro: Germinal matrix hemorrhage-intraventricular hemorrhage: pathogenesis and outcomes. Italian Journal of Pediatrics 2015 41(Suppl 1):A31 